\title{
La diplomatie du Pape François entre révolution et réactions
}

\author{
Blandine Chelini-Pont \\ Professeure d'histoire contemporaine et relations internationales \\ à l'Université d'Aix-Marseille.
}

Si l'on définit le terme révolution comme une rotation complète d'un corps mobile autour de son axe, il est possible, en filant la métaphore astrale, de qualifier François, premier pape sans chiffre et au prénom révélateur, de pape révolutionnaire. Élu pour redresser une Église en crise, ébranlée en profondeur par les scandales de ce qu'il appelle lui-même le cléricalisme ${ }^{1}$, en plus de la corruption et des abus sexuels, François a proposé un traitement radical en interne, mais aussi, à l'extérieur, une autre philosophie de la vie internationale, qui a d'abord emporté les suffrages, avant de provoquer, par ondes de choc successives, des réactions, des résistances et des critiques parfois virulentes, dont une véritable bronca au sein du catholicisme états-unien, presque au bord du schisme ${ }^{2}$. Car François a apporté un regard «sudiste », voire tiers-mondiste, aux désordres du monde. En conséquence, il a renversé la parole et l'action diplomatiques du Saint-Siège. Il a vu, depuis l'autre hémisphère et les périphéries de l'humanité, un monde en crise, couvert de plaies globales et interconnectées : scandale de la pauvreté, état de guerre endémique, globalisation de l'indifférence, destruction de l'écosystème, etc. François a ainsi prophétisé une nécessaire conversion du système économique, de la logique militaire, du traitement de la mobilité humaine et de l'exploitation de la nature, appelant la communauté internationale à se mobiliser pour réaliser cette conversion. En prime, il a invité les États à bâtir entre eux une vraie éthique relationnelle, fondée sur la recherche sincère du dialogue, de la confiance et de la négociation. Mais son appel a buté sur bien des critiques. Alarmant la communauté internationale du tragique et collectif désaxement des sociétés contemporaines concourant en prime à la destruction de l'écosystème, François propose une ligne programmatique apte à redresser l'orbite global, dont beaucoup contestent l'analyse.

\section{Désaxement sociétal : une présentation contestée des déséquilibres du monde}

Par ses médiations directes, celles de la secrétairerie d'État et de son réseau de 200 nonces, vicaires et délégués apostoliques auprès d'autant d'États et instances internationales ; par ses constantes visites apostoliques ${ }^{3}$ qui sont autant de temps de relations bilatérales qu'une communication globale ${ }^{4}$, François donne voix aux sans-voix, aux victimes et aux exclus qui subissent la violence de la pauvreté, la violence de la guerre et de l'insécurité terroriste, la

\footnotetext{
${ }^{1}$ Discours aux Congrégations générales, 7 mars 2013 ; Discours devant les membres de la Curie pour l'ouverture des fêtes de la Nativité, 22 décembre 2014, surnommé par la presse le « discours des 15 maladies » - de l'Église.

${ }^{2}$ Voir John Gehring, The Francis Effect. A Radical Pope's Challenge to the American Catholic Church, Lanham, Rowman \& Littlefield, 2017; et Nicolas Sénèze, Comment l'Amérique veut changer de pape, Paris, Bayard, 2019. 339 voyages apostoliques depuis 2013, en incluant l'année 2020, et une cinquantaine de pays visités dont 17 pays d'Europe, intra et extra-Union, 11 pays d'Amérique, sept pays d'Afrique dont l'Égypte et le Maroc, trois pays du Caucase, sept pays d'Asie, quatre pays du Moyen-Orient.

${ }^{4}$ Par le truchement des médias classiques mais également l'usage régulier des réseaux sociaux comme Twitter Facebook, Instagram, YouTube, du site d'information en ligne news.va et de la plate-forme jeunesse pope2you.
} 
violence des déplacements et des fermetures de frontières et la violence de la dégradation environnementale, laquelle a fait l'objet pour la première fois d'une doctrine très articulée ${ }^{5}$. À force d'encycliques, d'exhortations et de discours' ${ }^{6}$, dont l'Exhortation sociale Evangelii Gaudium (2013) sur la logique économique du profit et de la maximisation financière, creusant toujours plus les écarts de richesse, les inégalités ${ }^{7}$ et le pillage écologique, le pape fournit une analyse systémique d'un monde mal en point, où «l'idolâtrie de l'argent » et la politique de puissance fabriquent une «culture du déchet », d'exclusion sociale et humaine, de prédation - des biens, des personnes et des ressources - et de violence.

\section{Migrants et culture de guerre : une posture clivante}

Deux grands thèmes se dégagent de cette sombre description de l'état du monde : le rejet des migrants, recoupant la catégorie incertaine des réfugiés, et la culture de guerre. Les migrants, selon François, sont le non-objet d'une indifférence abyssale ou le prétexte d'une surenchère politique dangereuse, fondée sur des préjugés souvent raciaux. Son entier pontificat veut symboliser leur défense - commune à celle de tous les exclus -, à travers son déplacement à Lampedusa en juillet 2013, son discours devant le Congrès états-unien de septembre 2015, son déplacement à Ciudad Juárez en février 2016, suivi d'une visite en avril aux réfugiés de Lesbos et d'un retour en avion très controversé avec des réfugiés syriens musulmans. Ses appels sont en partie à l'origine de la politique d'ouverture menée par l'Allemagne durant la crise de 20152016, tout comme de celle, plus partielle, des executive orders présidentiels sous Barack Obama, après la mise en échec par le Congrès états-unien de sa grande loi sur l'immigration.

Le combat pour les migrants, relayé par les diocèses et une myriade d'associations catholiques nationales et internationales, est cependant la cause majeure de son affrontement avec Donald Trump, et un facteur de méfiance pour les catholiques (blancs) occidentaux, plus ou moins conservateurs, aux États-Unis et en Europe, travaillés par la défense de la civilisation chrétienne et le rejet de l'islam. Ces derniers votent contre l'ouverture de leurs frontières et pour le refus des immigrés; ils aident à l'avènement de partis conservateurs nationalistes en Pologne, en Hongrie, en Autriche ou en Italie - comme aux États-Unis -, et accusent François de trahison et d'islamophilie ${ }^{8}$.

L'autre grande bataille du pape François est celle de la culture de guerre endémique, «la troisième guerre mondiale en morceaux », avec ses multiples foyers, ses armements abondants et, au bout de sa logique, la course nucléaire. Son implication institutionnelle dans la politique onusienne d'interdiction des essais nucléaires, et plus récemment d'interdiction totale des armes

\footnotetext{
5 Sous le nom d'écologie intégrale, encyclique Laudato si' (2015). Voir également l'exhortation Querida Amazonia (2020), qui fait le lien entre écologie, respect des cultures et place des femmes.

${ }^{6}$. Discours au Parlement de l'Union européenne, discours au Conseil de l'Europe, 25 novembre 2014 ; Discours de Sarajevo, 6 juin 2015 ; Discours de politique générale aux Nations Unies, 25 septembre 2015 ; Discours devant le Congrès états-unien, 24 septembre 2015 ; Discours annuels (janvier) devant le corps diplomatique accrédité auprès du Saint-Siège.

${ }^{7}$ Laquelle lui a valu la réputation chez les catholiques nord-américains conservateurs d'être socialiste ou marxiste : "Rush Limbaugh lashes out at Francis : The "Evangelii Gaudium" is hypocritical and "pure Marxism" », La Stampa, 30 décembre 2019.

${ }^{8}$ Laurent Dandrieu, Église et immigration : le Grand malaise. Le pape et le suicide de la civilisation européenne, Paris, Les Presses de la Renaissance, 2017.
} 
atomiques (traité de 2017), se double d'une dénonciation de plus en plus bruyante de l'état de guerre, dont dernièrement son plaidoyer pour le Yémen dans un discours aux Émirats arabes unis. François s'est attaqué à la logique de dissuasion nucléaire, horreur éthique et monstruosité financière. Son appel au désarmement nucléaire total (Japon, novembre 20199), à la «paix désarmée », suit la logique qui l'a fait soutenir, en 2015, l'accord de Vienne sur le nucléaire iranien, puis critiquer le retrait états-unien de cet accord en 2018 et condamner les sanctions économiques qui ont suivi. Il explique aussi son inquiétude grandissante devant la détermination de Téhéran à reprendre son activité fissible - alors qu'il tente de maintenir l'Iran dans l'accord grâce à des échanges diplomatiques assez réguliers - et appelle également à une négociation urgente avec la Corée du Nord.

\section{Une critique de la politique militaire états-unienne?}

À ce propos, François semble empreint de grande méfiance vis-à-vis de la puissance militaire états-unienne et de son usage. Il a fait savoir sa réprobation de l'usage des drones et autres robots-tueurs par les États-Unis (2014) dans la guerre contre la terreur, sa réprobation de l'usage IA/robotique dans la guerre et de l'usage des bombardements aériens ciblés en Irak et Syrie ; il avait d'ailleurs personnellement contribué à empêcher les bombardements de représailles franco-américains en Syrie en 2013, sous B. Obama. De fait, une partie de l'incompréhension actuelle entre États-Unis et Saint-Siège se trouve, sans être clairement énoncée, dans la politique militaire états-unienne au Moyen-Orient - acceptée cependant le temps bref de la coalition internationale anti-Daech pour protéger les chrétiens des exactions de l'organisation terroriste -, et aujourd'hui dans le sens donné à la présence de l'Organisation du traité de l'Atlantique nord (OTAN) en Europe. Le Saint-Siège ne semble en effet pas considérer d'un si bon œil le maintien, l'élargissement et le renforcement financier récent de l'Alliance atlantique, ni l'extension de ses missions, en Europe et au-delà, surtout quand son usage n'a pas été requis par la communauté internationale.

\section{Critique du fossé libéral (et antirusse) européen?}

À l'inverse, il existe bien une nouvelle vision romaine de la paix en Europe, dans laquelle la Russie serait pleinement intégrée aux relations européennes, au nom d'une logique géographique et d'une matrice commune euro-chrétienne dont Moscou se revendique. Reçu trois fois par François (2013, 2015 et 2019), Vladimir Poutine est pris aux mots de son discours de 2013 à Valdaï : il s'y décrit comme le défenseur des valeurs chrétiennes et familiales face à un Occident en déclin moral, idée que François partage. L'Occident européen et l'Union font en effet l'objet d'une critique romaine ancienne mais désormais plus vigoureuse : critique de son libéralisme culturel et légal, qui cherche à s'ériger en modèle universel quand il s'enfermerait dans l'égoïsme et la confusion anthropologique (avortement, liberté sexuelle, biopolitique, mariage homosexuel, théorie du genre), critique de son atrophie spirituelle et de

\footnotetext{
${ }^{9}$ Provoquant la réaction négative de la France : Discours du Président Emmanuel Macron sur la stratégie de défense et de dissuasion, l’Ecole de guerre, Paris, 7 février 2020
} 
sa pente séculariste finalement intolérante aux croyances et à leur influence dans l'innervation sociale, critique enfin de son indifférence dramatique envers le sort des migrants.

Qui plus est, face à un Occident européen hostile, François semble croire au rôle stabilisateur de la Russie en Syrie, notamment quand elle est perçue comme 'modérant' les bombardements du régime syrien contre les « rebelles ». François a également affirmé que la Russie pouvait œuvrer de bien des façons à la paix mondiale, appelant l'Occident à faire son autocritique en Libye et évoquant les convergences sur les conflits du monde arabe. Sur la place légitime de la Russie, sur l'action contestable de l'OTAN ces dernières années, y compris dans sa stratégie d'encerclement russe, sur l'immoralité de la dissuasion nucléaire, la parole de François n'est pas prise au sérieux, ni par les Etats-Unis ni par la plupart des nations européennes, même si le débat sur la défense européenne face au désengagement américain en Europe semble faire bouger quelques lignes à l'Ouest.

\section{Redressement de l'orbite : l'éthique du dialogue et sa remise en question}

L'exemple européen offre finalement une autre clef de la révolution diplomatique de François. Le pape propose la prise à bras-le-corps par la communauté internationale de priorités globales dans une action concertée éthiquement fondée, que Jean-Baptiste Noé baptise « la diplomatie de miséricorde ${ }^{10}$. Mais il suggère également une éthique relationnelle entre États, fondée sur la recherche du dialogue et de la concorde. Or cette éthique de dialogue interétatique obéit chez lui à une logique continentale. La diplomatie globale en direction de la communauté internationale se double ainsi de focales régionales ou continentales, inspirées par les contextes culturels spécifiques, mais ces dernières ne sont pas toujours comprises ou considérées comme pertinentes.

\section{Un panaméricanisme pontifical}

La méthode de François est souvent définie comme multilatérale pour ses appels aux actions et décisions collectives de la communauté internationale, mais elle est aussi sensible à l'équilibre de paix sur les continents. Avec les Amériques, François pense unité, voire union, comme jadis les papes du $\mathrm{XX}^{\mathrm{e}}$ siècle pensaient celle de l'Europe. L'intense activité diplomatique qu'il a menée dans cette zone après son élection a cherché à «briser les murs » hérités de la guerre froide et de la période encore vivace des dictatures et des régimes marxistes, sur fond de domination états-unienne : en ce sens, sa médiation pour le retour des relations entre Cuba et les États-Unis (2014) a semblé fissurer le «mur des Caraïbes ». François a aussi cherché à faire tomber le mur des antagonismes politiques et des conflits intérieurs par des interventions conduites au Venezuela - plusieurs sous Hugo Chávez et Nicolás Maduro -, en Colombie - succès de la médiation catholique entre Forces armées révolutionnaires de Colombie (FARC) et gouvernement -, par des appels à la réconciliation nationale au Mexique, en Bolivie, au Salvador, y compris même par son récent silence dans la crise présidentielle au Venezuela, afin de ne pas contribuer au basculement dans le «bain de sang ». Le panaméricanisme pontifical

\footnotetext{
${ }^{10}$ Jean-Baptiste Noé, François le diplomate. La diplomatie de la miséricorde, Paris, Salvator, 2019.
} 
- et son appui au projet (lointain) d'une libre circulation concertée entre Américains - a rencontré un bon écho sous la présidence Obama dans son action centre et sud-américaine. Mais François se trouve à nouveau inaudible face à une administration Trump concentrée sur ses frontières, traquant ses migrants, boudant le sommet des Amériques, mettant fin aux accords commerciaux et distribuant sanctions économiques et commentaires menaçants envers ses voisins « socialistes », dont Cuba.

\section{En Asie, des petits pas très controversés}

Qui plus est, le scepticisme voire l'incompréhension que provoque la nouvelle Ostpolitik du Saint-Siège au sein des pays de l'OTAN, paraît presque minime face aux réactions vives que suscite sa politique asiatique de petits pas. Conscient de la croissance exponentielle des chrétiens dans cet espace tout comme des tensions qui s'y renforcent, François a pu visiter la Corée du Sud et le Japon. Pendant l'année 2018, quand la Corée du Nord a commencé son ouverture, il a d'ailleurs soutenu sa politique de rapprochement avec son voisin du Sud. Le pape a aussi répondu à l'invitation de Kim Jong-Un de venir en Corée du Nord - où la persécution religieuse atteint des sommets - sous la forme d'une délégation de la Communauté Sant'Edigio reçue à Pyongyang et se proposant d'être le lien entre les communautés chrétiennes des deux Corée. Cette main tendue a pu être taxée d'opportunisme déplacé, quand ce n'est pas de naïveté, sur les chances d'une normalisation coréenne. La même critique lui a été adressée face à l'accord semi-secret signé avec Pékin (septembre 2018) sur la nomination des évêques chinois, pourtant vécue à Rome comme une grande avancée, tout comme ses longues négociations avec le Viêtnam (août 2019), qui n'ont toujours pas abouti. L'accord chinois du Vatican est ainsi qualifié de capitulation et de braderie par le cardinal et archevêque émérite de Hong-Kong, Joseph Zen, qui regrette l'absence de soutien du pape à la défense désespérée du système démocratique par les Hong-Kongais et par des leaders majoritairement protestants.

\section{La Russie et l'Ukraine, marches de la paix religieuse}

La politique de main tendue envers les « démocratures » russe et chinoise, pour exemplaire qu'elle soit d'une application de l'éthique de confiance, a aussi en arrière-plan une intention religieuse. Il s'agit là d'une dernière intuition propre à la pastorale du pape François : selon lui, la paix entre les peuples ne peut se consolider sans tolérance ni liberté religieuse, et il espère, à force de mains tendues, rendre effectif le retournement des cœurs et des législations. Cette dimension est en quelque sorte la propre contribution de l'Église catholique à la pacification du monde. Ainsi la paix religieuse est-elle la grande ambition, la grande vision du pape, une vision ouverte, qui aspire à dépasser le « choc des civilisations » et veut s'appuyer sur l'objectif de la coexistence croyante comme ciment le plus efficace de la paix universelle. C'est pour cette raison qu'il n'a pas désavoué la Russie dans le cadre de la crise ukrainienne, tout comme il n'a pas cédé à la demande de l'Église uniate d'Ukraine de devenir un patriarcat, ou encore qu'il n'a pas commenté la naissance d'une Église orthodoxe d'Ukraine autocéphale que le patriarcat de Constantinople avait fini par accepter, provoquant de facto une rupture avec Moscou. L'enjeu d'une intégration de l'orthodoxie russe en lieu et place de son irrédentisme à la communion œcuménique, est un espoir immense pour le Vatican et de ce point de vue, la rencontre entre 
François et Cyrille de Moscou à La Havane en mars 2019 a été historique. Et cette approche a vocation à s'étendre : elle s'est notamment traduite, en Asie, par des déplacements inédits dans les pays musulmans et bouddhistes, afin d'y défendre la cause des minorités chrétiennes et musulmanes maltraitées voire persécutées (Sri Lanka, Bangladesh, Birmanie, Thaïlande).

\section{Se démarquer de la poussée évangélique en Afrique et au Moyen-Orient}

Mais c'est en Afrique et dans le monde arabe que l'objectif de la paix religieuse prend toute son ampleur. La stratégie interreligieuse de l'Église sur le continent y a doublé sa traditionnelle défense de la démocratie, contre les présidences à vie, pour le respect de l'alternance démocratique (Bénin, Sénégal, République démocratique du Congo, Togo, Cameroun), pour la fin de la corruption électorale (Kenya) et la mise en place d'un vrai pluralisme politique (Tanzanie). La Communauté de Sant'Egidio et les instances interreligieuses comme Religions for Peace ont été remarquablement efficaces à nouer le dialogue dans les guerres civiles, à chercher des solutions entre acteurs locaux ou à freiner les conflits intercommunautaires (Mozambique, Soudan du Sud, Tchad, Centrafrique, Burkina Faso-Niger). Néanmoins, la forte progression évangélique, pentecôtiste et désormais celle des mouvements de prospérité freine la politique de collaboration religieuse panafricaine. Les Eglises de ces mouvances sont à la fois très prosélytes et ne sont pas du tout favorables au dialogue islamo-chrétien. Se distinguer des Eglises évangéliques constitue donc un enjeu parallèle du dialogue islamo-chrétien mené par l'Église catholique. Quand ces dernières déploient leurs sites Internet et leurs chaînes de télévision de l'Afrique jusqu'au cœur du monde arabe, où elles pratiquent un prosélytisme décomplexé, le Saint-Siège « interdit » tout prosélytisme envers les musulmans et, au MoyenOrient, il préfère défendre à la fois les droits des communautés chrétiennes orientales, la présence éducative et médicale des ordres et organisations catholiques, et surtout, plus récemment, l'aide étendue aux réfugiés des conflits syriens et irakiens.

La reprise du dialogue islamo-chrétien est, en effet, redevenue une priorité avec François, après sa mise en sommeil sous Benoît XVI. Le pape a multiplié les déclarations fraternelles et poussé aux rencontres diplomatiques : le cardinal Tauran auprès de la Ligue islamique mondiale en avril 2018, lui-même auprès d'Ahmed Al-Tayeb, grand imam de l'Université Al-Ahzar en Égypte (avril 2017), et récemment aux Émirats arabes unis, où il a rencontré à nouveau A. AlTayeb, puis le Conseil des sages musulmans et le prince héritier d'Abou Dhabi, qu'il a félicité sur la tolérance religieuse de son émirat (février 2019). Reçu avec tous les honneurs au Maroc, en mars 2019, François a visité 1'Institut de Formation des imams du Royaume et célébré une messe à Rabat auprès de fidèles très majoritairement migrants ou immigrés.

\section{Israël-Palestine : le changement dans la continuité}

C'est donc tout autant pour défendre ses intérêts, les intérêts des chrétiens palestiniens et l'enjeu de la paix religieuse avec les musulmans que le Saint-Siège maintient sa position dans le conflit israélo-palestinien. François n'a pas innové sur cette question : le Saint-Siège défend depuis longtemps l'internationalisation de Jérusalem, qu'Israël n'entend pas admettre, et les droits des Palestiniens à avoir un État et un territoire, notamment face à la colonisation de peuplement juif. En guise d'exemple à suivre, François a signé un traité bilatéral de nature internationale 
avec l'Autorité palestinienne en 2015 sur la présence de l'Église catholique en Palestine, qui actait de ce fait sa reconnaissance d'un État palestinien «selon le droit international». Son absence de commentaire précédée d'avertissements vis-à-vis du plan de paix annoncé puis présenté par D. Trump en janvier 2020 en a constitué une suite logique, tandis que les Evangéliques présents en Israël, souvent d'origine états-unienne, sont, pour leur part, favorables à l'extension de l'État hébreu à la frontière du Jourdain, et défendent des positions sionistes derrière le puissant réseau de l'International Fellowship of Christians and Jews.

La vision de la paix à construire du pape François ne fait pas l'unanimité - particulièrement auprès des États-Unis de D. Trump - ni dans sa dimension globale ni dans ses dimensions continentales, en ce qu'elle s'oppose assez directement à la position isolationniste, antiécologique, financièrement et militairement défensive des États-Unis contemporains. François est également dubitatif sur l'action apparemment plus universelle de l'Europe. De même, si François cherche à construire les fondements d'une culture globale de la paix, son prisme sudiste et non occidental, son appel à changer de système économique, à ouvrir les portes aux migrants, à expérimenter l'union interreligieuse et à construire une paix désarmée ont aussi provoqué des tensions, des incompréhensions et une forme de méfiance dans son jugement chez une partie des catholiques nord-américains et européens, mais également parmi les Evangéliques. Il n'est pas dit non plus que son appel au dialogue islamo-chrétien soit entendu de tous les musulmans. Toutes ces remises en question affaiblissent certes la révolution de François. Elles ne peuvent toutefois empêcher le constat, qu'avec ce pape, c'est finalement l'axe de rotation de l'Église catholique qui s'est tout entier modifié pour tenter de changer celui de la planète. 